
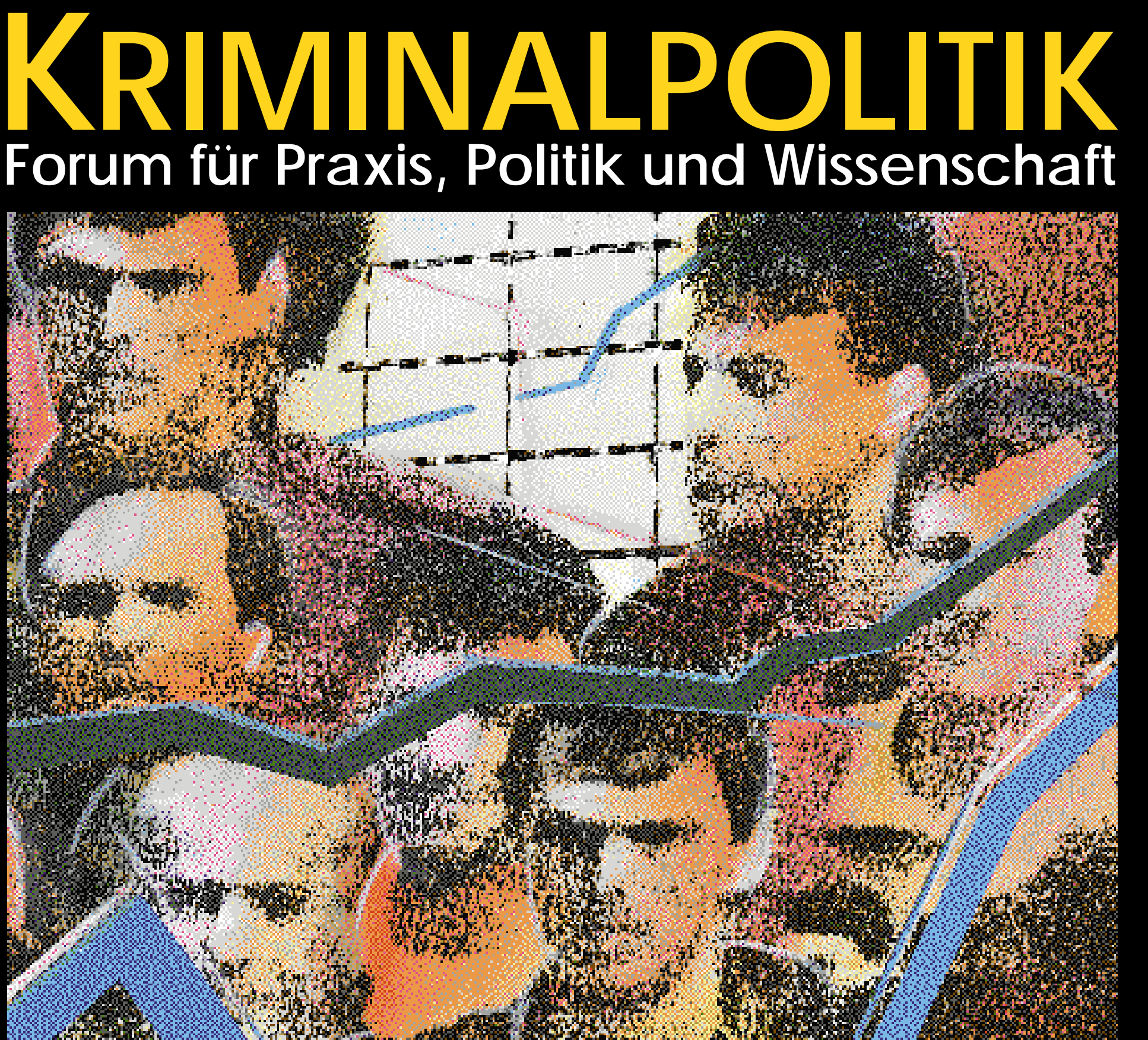

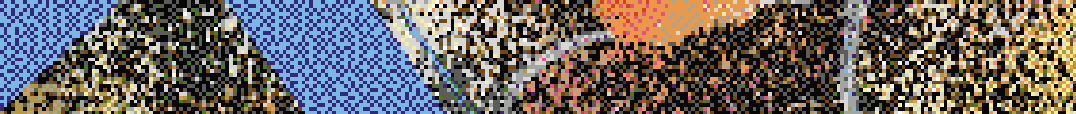

4 is

6ros

is

Wre

civis

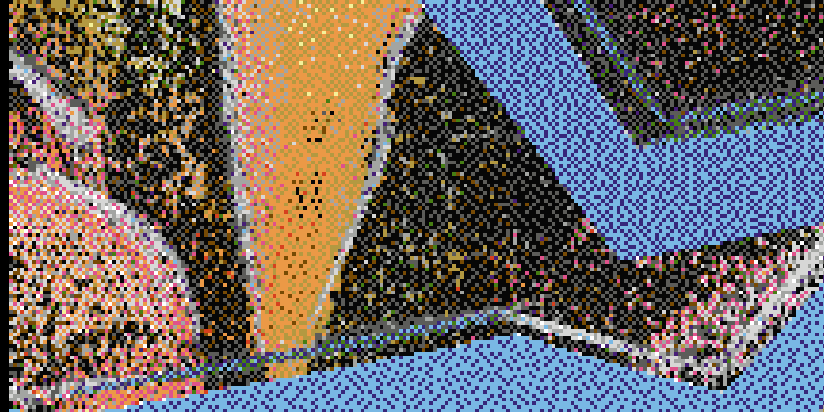
is

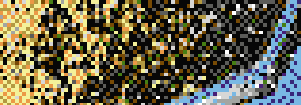

$x^{2}$ ist

(3)

\title{
DER DATEN-SCHWINDEL
}

Kriminalstatistik: Interessen, Strategien und Legitimationen - Texte von Pfeiffer, Wetzels, Jehle und Boers 


\section{Schnellschüsse...}

$\mathrm{K}$

aum ein Bereich der Politik wird derzeit in der Öffentlichkeit so heftig diskutiert und strapaziert wie die Kriminalpolitik. Regierung und Opposition scheinen sich gegenseitig übertreffen zu wollen. Deutschland im Super-Wahljahr, da gilt es, die Law-and-Order-Muskeln spielen zu lassen - auch wenn Bürgerrechte dabei Schaden nehmen. Das Zauberwort heißt »Organisierte Kriminalität«. Immer neue Gesetzesentwürfe werden vorgelegt. Aktuell: Ein von der Regierungskoalition geplantes »Verbrechensbekämpfungsgesetz « und (die Sozialdemokraten wollen auch Stärke demonstrieren) der SPD-Entwurf für ein zweites Gesetz zur Bekämpfung der organisierten Kriminalität.

Eine breite Allianz der Ablehnung hat sich formiert: Die Bundesrechtsanwaltkammer (BRAK), der Deutsche Anwaltsverein (DAV) und die Strafverteidigervereinigungen lehnen beide Entwürfe ebenso entschieden ab wie die Neue Richtervereinigung sowie die in der ÖTV organisierten Richter und Staatsanwälte.Viele Vorschläge seien »absolute Schnellschüsse«, so der BRAK-Geschäftsführer Johnigk. »Gesetzesänderungen, bei denen es um Eingriffe in Grund- und Bürgerrechte geht, sollten nicht im Superwahljahr erfolgen, in dem eine sachgerechte, emotionsfreie Beratung nicht möglich ist «, so dessen Meinung. Rainer Brüssow, Geschäftsführer des Anwaltsverein, kritisiert, daß nicht abgewartet werde, wie sich die verabschiedeten Gesetze - das Geldwäschegesetz, das Rechtspflegeentlastungsgesetz sowie das erste Gesetz zur Bekämpfung der organisierten Kriminalität - auswirken. Es scheint, als käme es derzeit freilich nur noch darauf an, im Wettbewerb um Wählerstimmen ständig neue Gesetze zu präsentieren. Die vorhandenen werden dabei gerne übersehen... Dazu paßt, daß in diesen Zeiten die Bürger wiederholt mit Nachrichten über einen dramatischen Anstieg der Kriminalität aufgeschreckt werden. Keine Frage: Mit Kriminalpolitk wird Politik gemacht - und Statistiken liefern dazu häufig genug fragwürdiges Material. In diesem Heft geht es um den Miß- brauch und die Fehlinterpretationen - und um ein Plädoyer für eine andere Kriminalstatistik. Unser Titel ab Seite 32.

Noch etwas Erfreuliches: Oliver Weiss, Karikaturist aus Grassau in Bayern, aufmerksamen NK-Lesern durch seine ebenso humorvollen wie tiefsinnigen

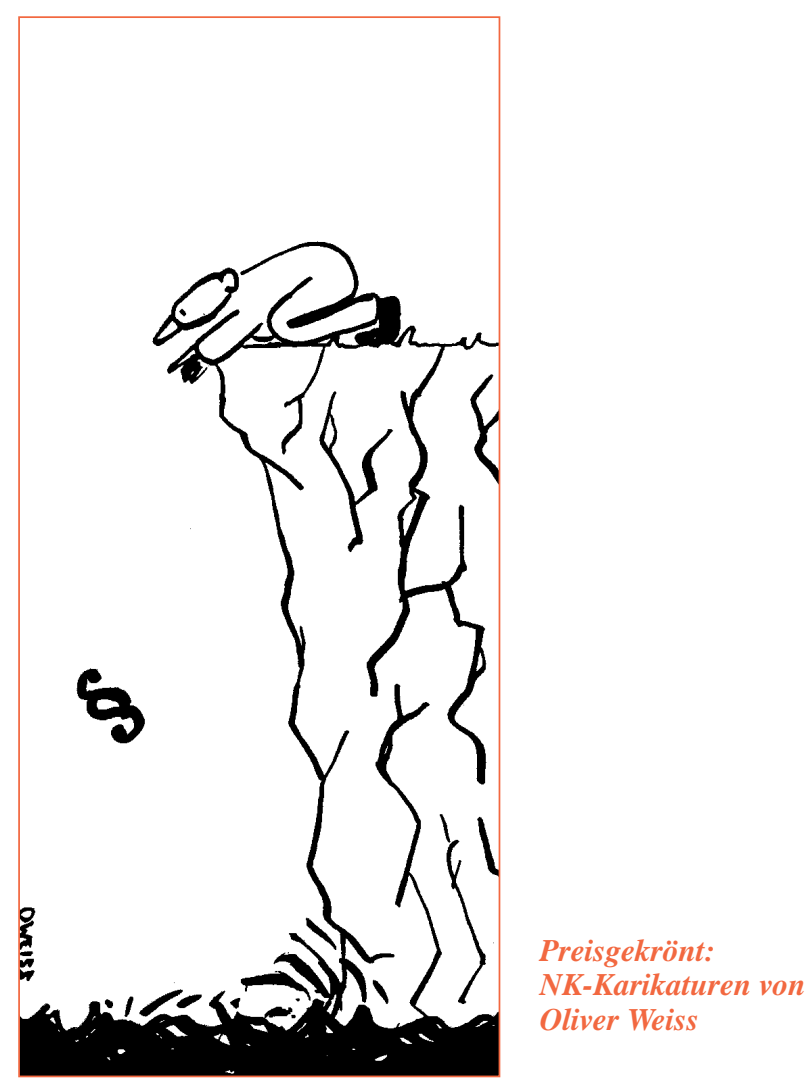

Zeichnungen bekannt, bekam öffentliche Anerkennung: Beim ÖTV-Wettbewerb »Gegen Rassismus « gewann er einen Hauptpreis, im internationalen polnischen Karikatur-Wettbewerb »Satyrykon 94 « gab's für ihn einen Sonderpreis.

Herzlichen Glückwunsch! Neue Karikaturen von Oliver Weiss finden Sie auch in diesem Heft.

Ihnen wünsche ich wie immer gute Fachlektüre

\section{Ihr Helmut Ortner}




\section{Der Daten-Schwindel}

Krimina Istatistiken sind nicht nur Produkte der Kriminalpolitik - mit innen wird auch Politik gemacht. Es geht um Interessen, Strategien und Legitimationen. Unsere Titelbeiträge von Jörg-Martin Jehle, Klaus Boers, Christian Pfeiffer und Peter Wetzels ab Seite 20

\section{》Ein guter Minister ist immer unzufrieden ...« \\ Strafjustiz, Strafvollzug und \\ Straffälligenhilfe in den neuen Bundesländern. Keine Wende nach der Wende? Inge $G$ ünther sprach über kriminalpolitische Probleme und Perspektiven mit dem brandenburgischen Justiz mi- nister Hans 0 tto Bräutigam. ab Seite 16}
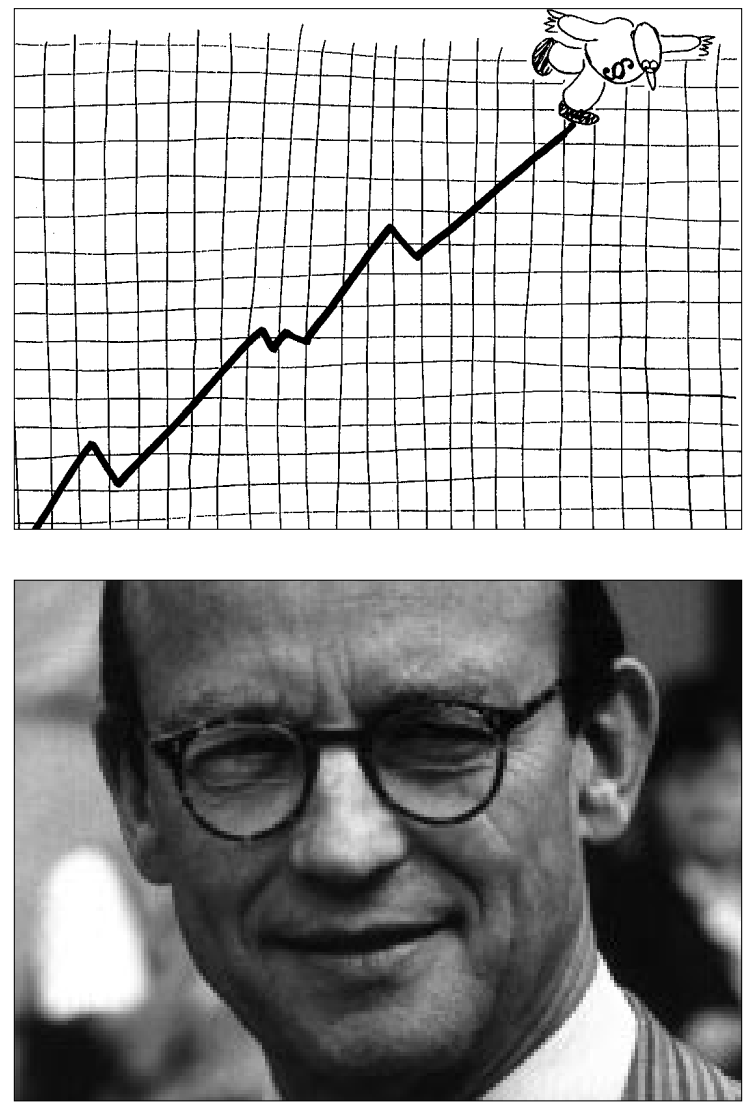

\section{Drogen und Verfassungswidrigkeit}

Das Bundesverfassungsgericht hat entschieden, da B

Handel mit Haschisch in »nicht geringer M enge mit $^{2}$ einer M indeststrafe von einem Jahr geahndet werden kann. Die Diskussion strafbesetzter Drogenprohibition ist damit jedoch nicht am Ende. Ein Beitrag von Ralf Hohmann und Holger Matt ab Seite $\mathbf{4 0}$

\section{Stanley Cohen - Der Engagierte}

Er gilt als Vordenker einer kritischen Kriminologie,

lehrte als Soziologe an verschiedenen Universitä ten und lebt heute in Jerusalem: Stanley Cohen.

N K-A utorin Ruth Herz hat ihn dort besucht. Ihr Portrait

ab Seite 50
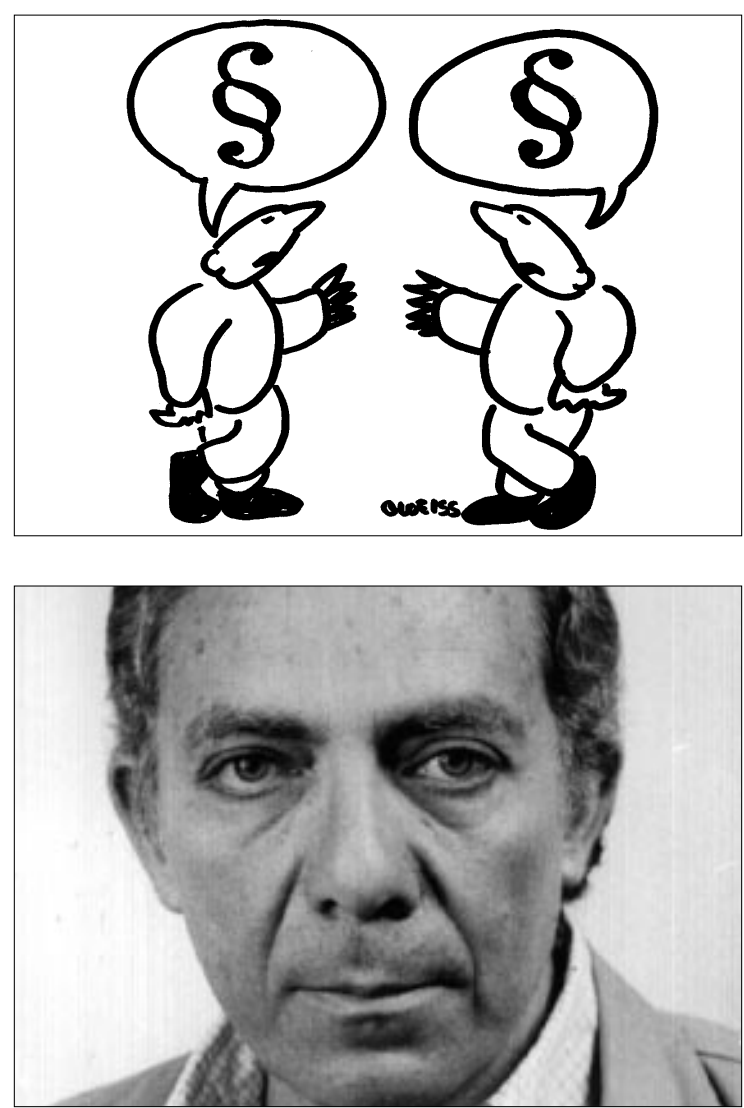

\section{TITEL}

\section{Der Daten-Schwindel}

Kriminalstatistiken: Interessen, Strategien und Legitimationen

Plä doyer für bessere Kriminalstatistiken

Jörg-Martin Jehle

Kriminalität und Krimina litätsfurcht

im sozialen Umbruch

Klaus Boers

»Die Explosion des Verbrechens? «

Christian Pfeiffer/ Peter W etzels

\section{MAGAZIN}

Tagungsbericht: Zur Kooperation verurteilt? 6 - Anhörung: Gegen Lebenslänglich 7 - Strafvollzug: Falsches Signal? 8 - Justizvollzug: Schulausbildung 9 • Präventionsräte: Modell mit Perspektive? 10 • Ö sterreich: N otwendige Reform 12

\section{MEINUNG}

W ehrhafte Toleranz

Thomas Schmid

\section{BEITRA G}

Drogen und Verfassungswidrigkeit

Ralf Hohmann/ Holger Matt

Paternalismus und M oralismus

Jean-Claude W olf/ Detlef Leber

\section{PORTRAIT}

Der Engagierte - Stanley Cohen

Ein Portrait von Ruth Herz

\section{RUBRIKEN}

Editorial

Terminal

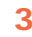

Praxis

Bücher

Impressum

56

58

\section{EIN HEFTER}

Kriminalsoziologische Bibliografie

in der Heftmitte

Beilagenhinweis: Dieser A usgabe liegt ein Prospekt der $\mathrm{N}$ omos Verlagsgesellschaft bei. W ir bitten freundlichst um Beachtung. 